\title{
IC PACKAGING TECHNOLOGY FOR ELECTRONIC WATCHES
}

\author{
YOSHIO IINUMA \\ Citizen Watch Co., Ltd., Technical Laboratory, Saitama, Japan
}

\begin{abstract}
IC-Quartz watches have been manufactured since the appearance of CMOS-LSI about seven years ago. Nowadays, the outside view of the quartz watch is the same as that of a mechanical watch through minimizing electronic parts such as battery, crystal oscillator, capacitor, and IC packaging volume. Recently, IC packaging technology for electronic wrist watches (both the analog quartz watch and digital quartz watch) has made a step foward. In Japan, some watch makers adopt a film carrier assembly technique for producing thin and low-priced watches. They are using 3 layers and $35 \mathrm{~mm}$ wide film which fits very well the substrate of the quartz watch, especially the digital quartz watch.

The Citizen Watch Co., Ltd. started an investigation as to which method was suitable for IC package for watches in 1975 and adopted a flip chip technique as being the most suitable for analog quartz watches. The flip chip technique will allow the fabrication of a very small and thin package. Important characteristics of the technique permit design flexibility and high density packaging of the ICs. In this paper, a flip chip technique using a Printed Circuit Board as a substrate is described.
\end{abstract}

\section{INTRODUCTION}

There are several general requirements about IC packaging technique for a watch, such as miniaturization of packaging volume, inexpensiveness, reliability, design flexibility and production flexibility. In the case of the watch module, the most important requirement is miniaturization of packaging volume. It is not necessary to consider the dissipation of heat because of the low power consumption, nor consider standardization because of using a specifically designed CMOS-LSI and substrate.

In Table I, three bonding methods - wire bonding, face down bonding (Flip Chip) and tape automated bonding (Mini Mod) - are compared regarding conformance with the requirements specified above for both analog and digital quartz watches.

Wire bonding technique provides an IC package for all quartz watches, but it is difficult for a flip chip or mini mod technique to provide an IC package for all quartz watches. The flip chip technique is not suited for bonding the large-sized LSI required for digital watches. On the other hand, the mini mod technique is unsuited for a high density substrate, because a double-sided film carrier is very expensive compared with single-sided film.

Three years ago, the Citizen Watch Co. Ltd. started the production of analog quartz watches, especially for
TABLE I

Comparison of three bonding methods $(\mathrm{F}=$ Fair $; \mathrm{G}=\mathrm{Good}$; $\mathrm{E}=$ Excellent).

\begin{tabular}{|c|c|c|c|}
\hline Item & Wire & FC & $\mathrm{MM}^{\mathrm{a}}$ \\
\hline Miniaturization & 1 & $1 / 2-1 / 3$ & $1 / 2-1 / 3$ \\
\hline Cost $^{b}$ & 1 & 0.6 & 0.4 \\
\hline Reliability & G & $\mathrm{E}$ & $\mathrm{E}$ \\
\hline Design flexibility & G & $\mathrm{E}$ & $\mathbf{F}$ \\
\hline Production flexibility & $\mathrm{E}$ & $\mathbf{G}$ & $\mathbf{F}$ \\
\hline Analog quartz watch & $\mathbf{F}$ & $\mathrm{E}$ & G \\
\hline Digital quartz watch ${ }^{\mathrm{d}}$ & G & $\mathbf{F}$ & $\mathrm{E}$ \\
\hline
\end{tabular}

${ }^{a} 35 \mathrm{~mm}$ film carrier (3 layers).

${ }^{b}$ Production volume 100 thousand/month. Compared with assembly cost including bumping cost, not including chip and material costs.

${ }^{c}$ Using $2 \mathrm{~mm}$ chip, $10-15$ bonding pads.

${ }^{d}$ Using 4-6 mm chip, 40-80 bonding pads.

ladies, using the flip chip technique. Solder bumped CMOS-LSIs are automatically bonded on to Printed Circuit Boards (PCB). An epoxy resin is provided which fills the gap between the chip and the PCB in order to reduce the thickness to the minimum. As a result, the thickness is reduced by two-thirds and the quality is better than the previous technique of wire bonding. The PCB is good as a substrate for the electronic watch, because of its mechanical strength 
and the possibility of fine pattern and pattern location accuracy. The PCB is gold plated, and an interconnection pattern is formed for the purpose of preventing overwetting of solder bumps in the pattern.

The difference in co-efficient of thermal expansion between a CMOS-LSI chip and a PCB is a fundamental problem. In order to resolve this problem, the distance between the chip and the PCB after face down bonding is controlled. The size of solder bumps and the interconnection pattern of the PCB are decided so that the distance between the chip and the board is always more than the specified minimum value.

\section{EXPERIMENTAL PROCEDURE AND RESULTS}

\subsection{Bonding}

The substrate for an analog quartz watch is shown in Figure 1. The substrate material is polyimide glass laminated PCB. The PCB is double-sided and gold-plated, and has a thickness of about 350 microns.

The interconnection pattern where a CMOS-LSI is bonded face down is formed as shown in Figure 2. The width is $80-100$ microns with \pm 15 microns uniformity. This particular pattern is not spaced enough to prevent overwetting of solder bumps to the pattern; however, if bonding conditions - time, temperature and others are appropriately controlled, it is easy to get a satisfactory face down bonding, as shown in Figure 3.

10 bump LSI die (Die size is $2 \mathrm{~mm}$ ) have been face-down-bonded onto PCBs. The adhesive strength (f) of the LSI depends on the distance between the chip and the PCB as shown in Figure 4.

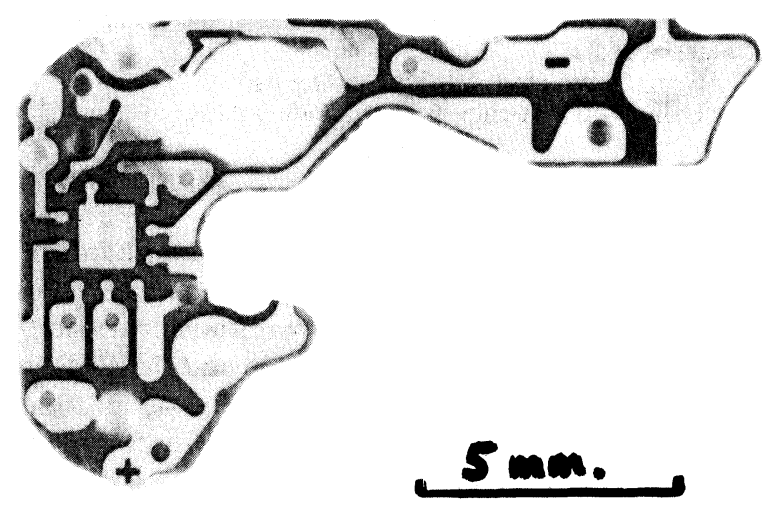

FIGURE 1 PCB for analog quartz watch.

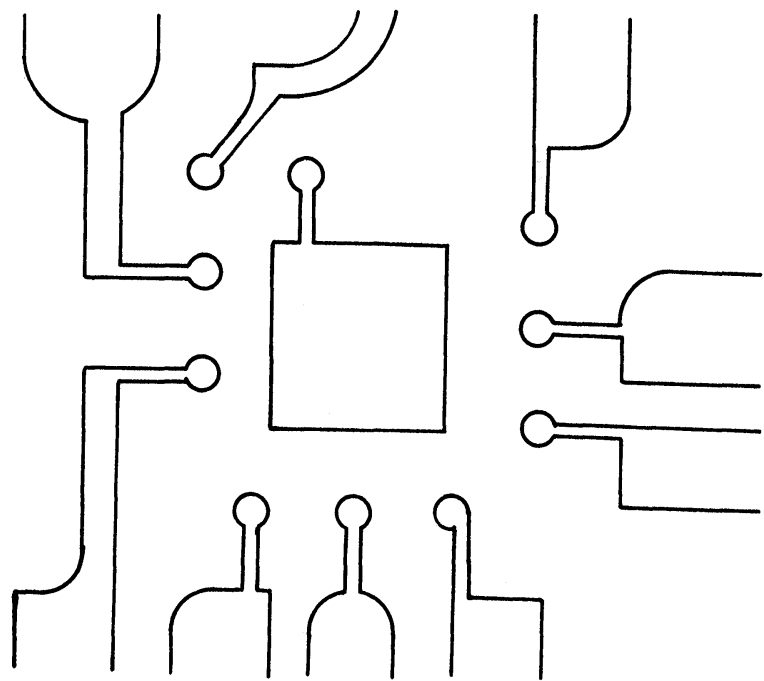

FIGURE 2 Interconnection pattern (minimum time width $80 \mu \mathrm{m})$.

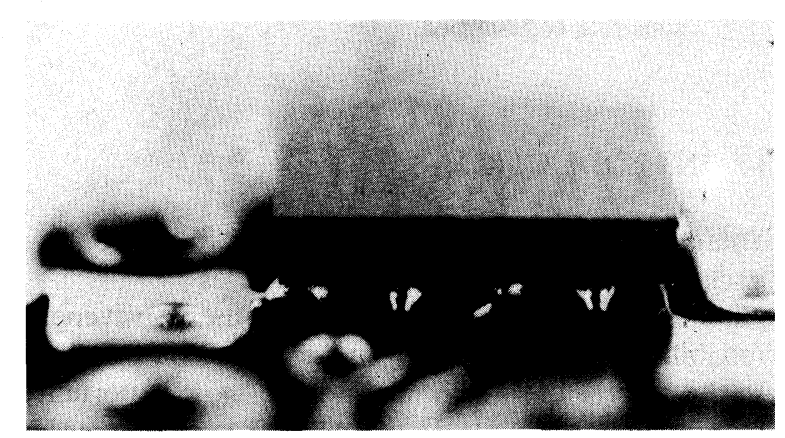

FIGURE 3 Face down bonding of device (die size $3 \mathrm{~mm}$ side).

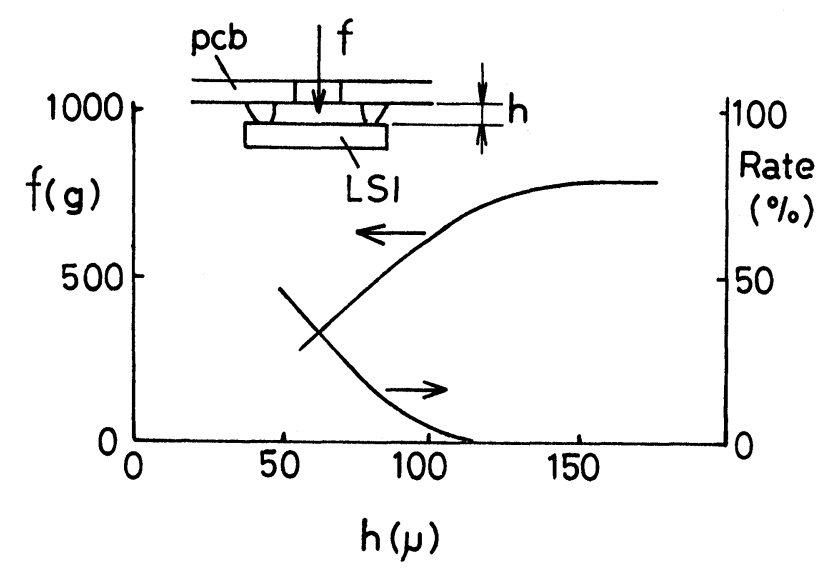

FIGURE 4 Adhesive strength (f) and the rate of undesirable breaking mode to the distance (h). 
The distance (h) depends on the size of the solder bumps. The adhesive strength is 800 grams $(80$ grams per bump) in the case of the distance (h) being more than 150 microns. The rate of the undesirable breaking mode - (silicon under the solder bump is destroyed as shown in Figure 5 - in consequence of heat stress which occurs when the LSI is bonded face down to the PCB), also depends on the distance (h) and is indicated in Figure 4.

The devices were tested after encapsulation by examining the bonding after the environmental and mechanical tests. As a result, if the distance (h) exceeds 80 microns, the device has sufficient marketable quality. In actual production the size of solder bumps is decided so that the distance (h) is kept at more than 100 microns after face down bonding, where the die size is about $2 \mathrm{~mm}$.

Solder bumps CMOS-LSI were automatically bonded on the PCB by mechanical location adjustment. Bonding speeds were 1000 to 1200 dies per hour. The pattern width accuracy of the PCB was \pm 15 microns and the pattern location accuracy of the basic hole was \pm 40 microns. Such precision was a satisfactory level for substrate for a wrist watch. A minimum quantity of flux which was required in order to get a stable bonding is supplied by a dispenser.

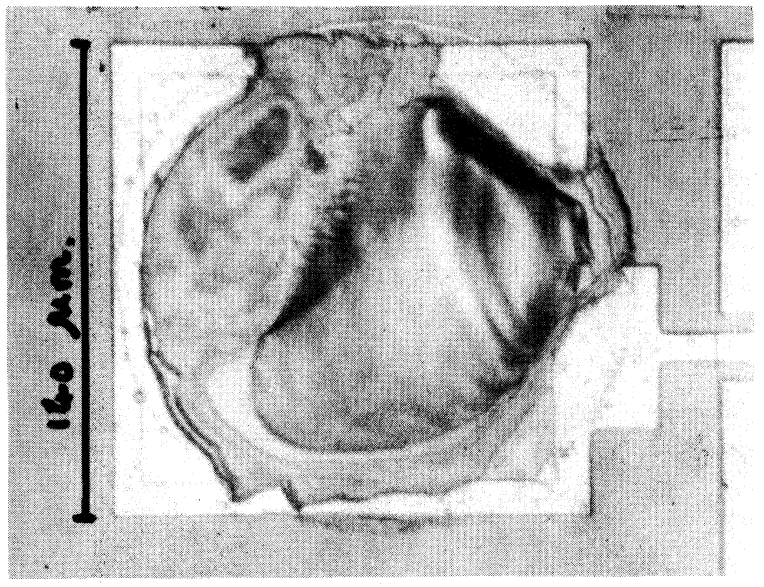

FIGURE 5 Undesirable breaking mode.

\subsection{Encapsulation}

With regard to a wrist watch substrate the encapsulation volume is always kept to a minimum. Diagrams of some sample encapsulations are shown in Figure 6, which shows the actual dimensions of the substrate for an analog quartz watch.

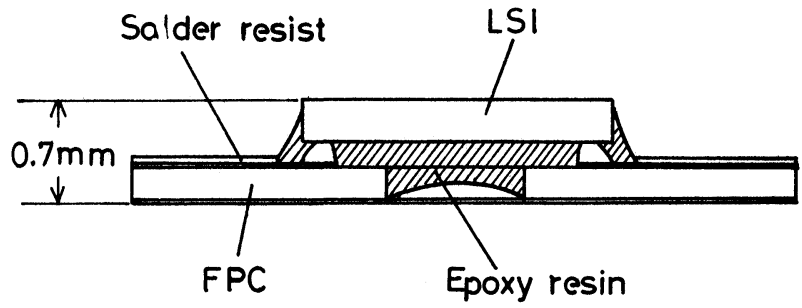

Type A

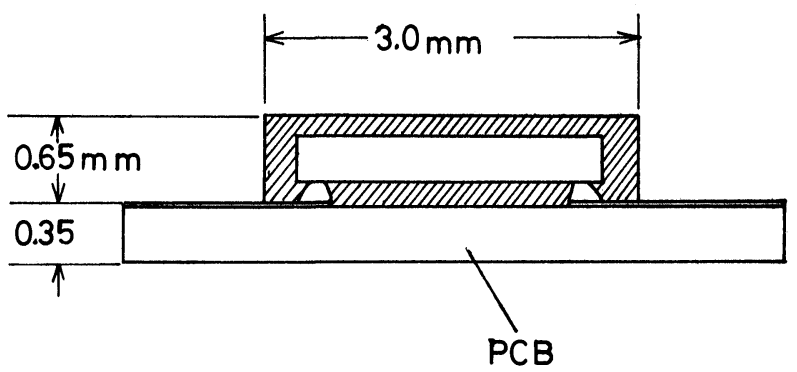

Type B
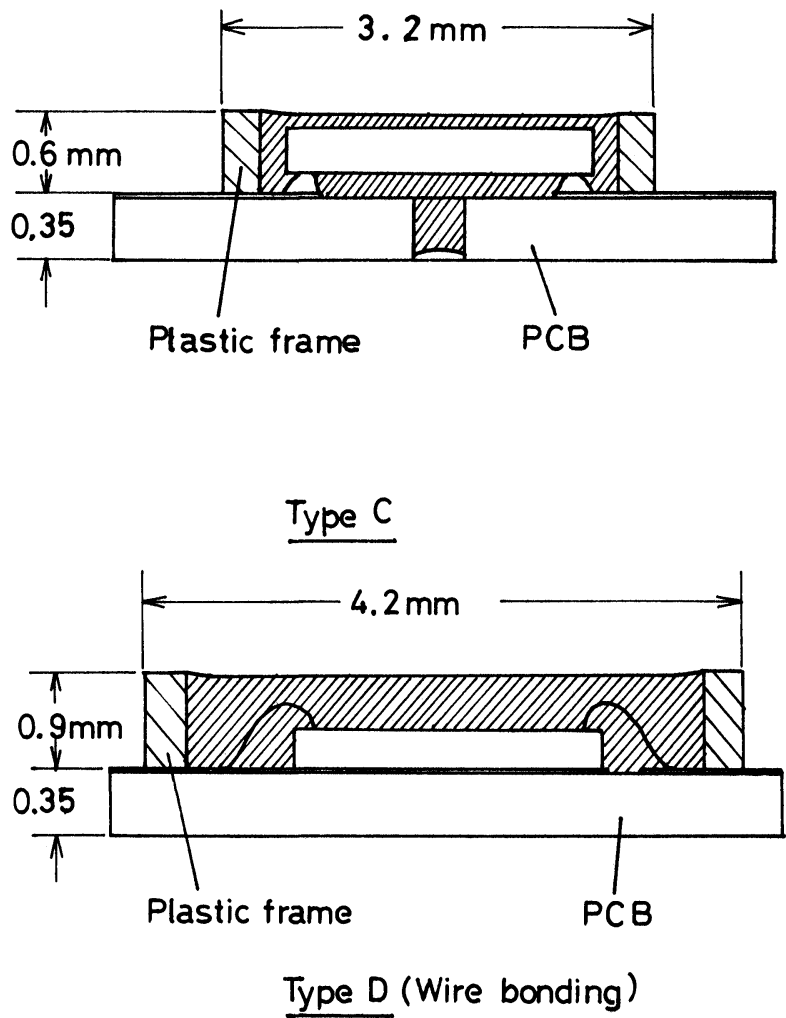

FIGURE 6 Samples after encapsulation. 
In example, Type A a drop of epoxy resin through the hole in the PCB fills the gap between the chip and the PCB. Solder resist prevents the spread of the epoxy resin. If the LSI is sensitive to light, the LSI is encapsulated as shown in Figure 6, Type B using transfer moulding. In Types $\mathrm{C}$ and $\mathrm{D}$, the plastic frame which is normally supplied around the LSI and the epoxy resin is provided by potting. In all of the samples (Types A, B and C) above, the packaging volume is reduced. The packaging volume of Type $A$ is reduced to one-third of that of Type D (wire bonding) provided that the size of the die is the same. The above three types (Types A, B and C) are similar in reliability to each other and the reliability is rather higher than conventional watch substrates, like Type $D$, shown in Figure 6. After encapsulation of the LSI, the crystal oscillator, trimmer condenser and the other parts are soldered onto the PCB.

\subsection{Reliability}

The substrates after encapsulation were tested under the following environmental conditions:

1) Temp. cycles $-45^{\circ}$ to $125^{\circ} \mathrm{C} \quad 100$ cycles

2) Humidity $65^{\circ} \mathrm{C} 95 \% \mathrm{RH} \quad 1000$ hours

3) High temp. storage $125^{\circ} \mathrm{C} \quad 1000$ hours

4) Biased high temp. life $65^{\circ} \mathrm{C} 1.8 \mathrm{~V} 100$ hours

The substrates were also tested in a completed watch using mechanical shock and vibration.

The rate of failure occurrence in relationship to the number of temperature cycles is shown in Figure 7. It can be seen that the flip chip technique shows considerable improvement over wire bonding.

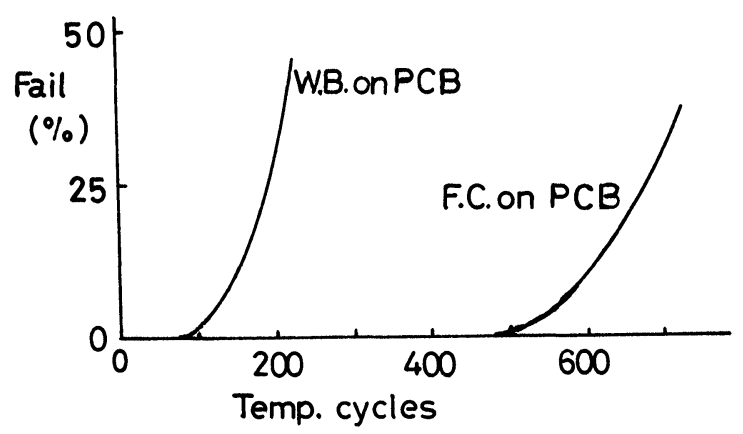

FIGURE 7 Failure rate vs. temperature cycles

\subsection{Application}

The Citizen Watch Co. Ltd. has developed an extremely thin analog quartz watch - the Caliber $790-$ which was the first analog quartz watch with a movement less than $1 \mathrm{~mm}$ thick in the world; the movement thickness of the Caliber 790 is $0.98 \mathrm{~mm}$, and with thin mechanical and other electrical parts, and a thin LSI package, as shown in Figure 6A, the total thickness including the watch case is $3 \mathrm{~mm}$ using a 0.9 thick battery. The completed substrate and movement of the Caliber 790 is shown in Figure 8. The substrate material is 5 mils polymide laminated on both sides with copper.

Some completed substrates of other analog quartz watches are shown in Figure 9.

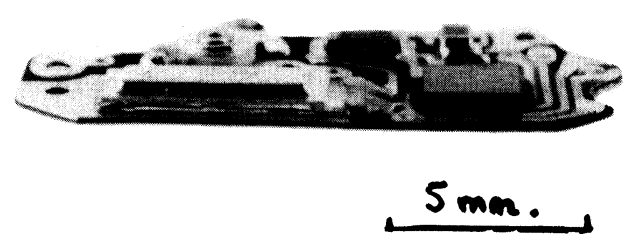

Substrate

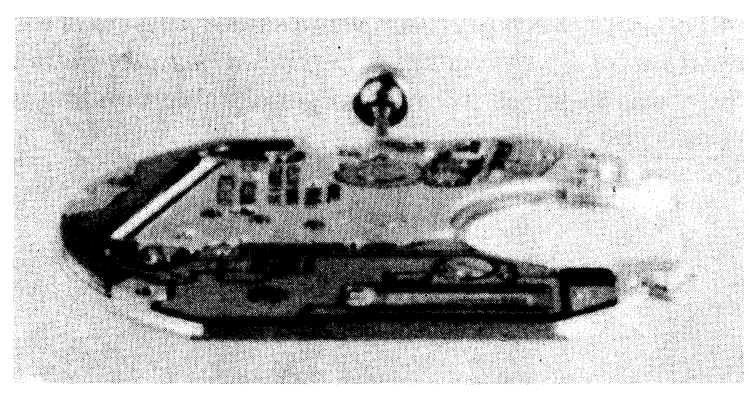

Movement

FIGURE 8 Extremely thin watch - Cal. 790.

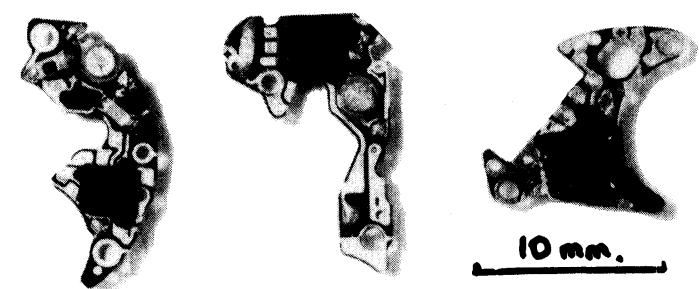

FIGURE 9 Some completed substrates.

\section{CONCLUSION}

A flip chip technique has contributed to the miniaturization of an IC packaging volume for an 
analog quartz watch. The whole production system of the technique except the bonding is almost similar to that of auto wire bonding, so it is very easy to change the IC packaging method from auto wire bonding to face down bonding.

Initially, the flip chip technique was applied to analog quartz watches. If the structure of the solder bump is investigated and the distance between the chip and the PCB is controlled, the flip chip technique will be able to be applied to large sized LSI, for example, $5 \mathrm{~mm}$ and 60 pads. Thus the flip chip technique will be an effective means for constructing quartz watch circuits. In the future flip chip and mini-mod techniques will be tested further to overcome the problems of applying IC packaging techniques to quartz watches in actual production conditions.

\section{ACKNOWLEDGEMENTS}

I wish to thank all of the people working in the same group as myself at the Citizen Watch Co., Ltd. Technical Laboratory for their technical assistance and numerous discussions. 

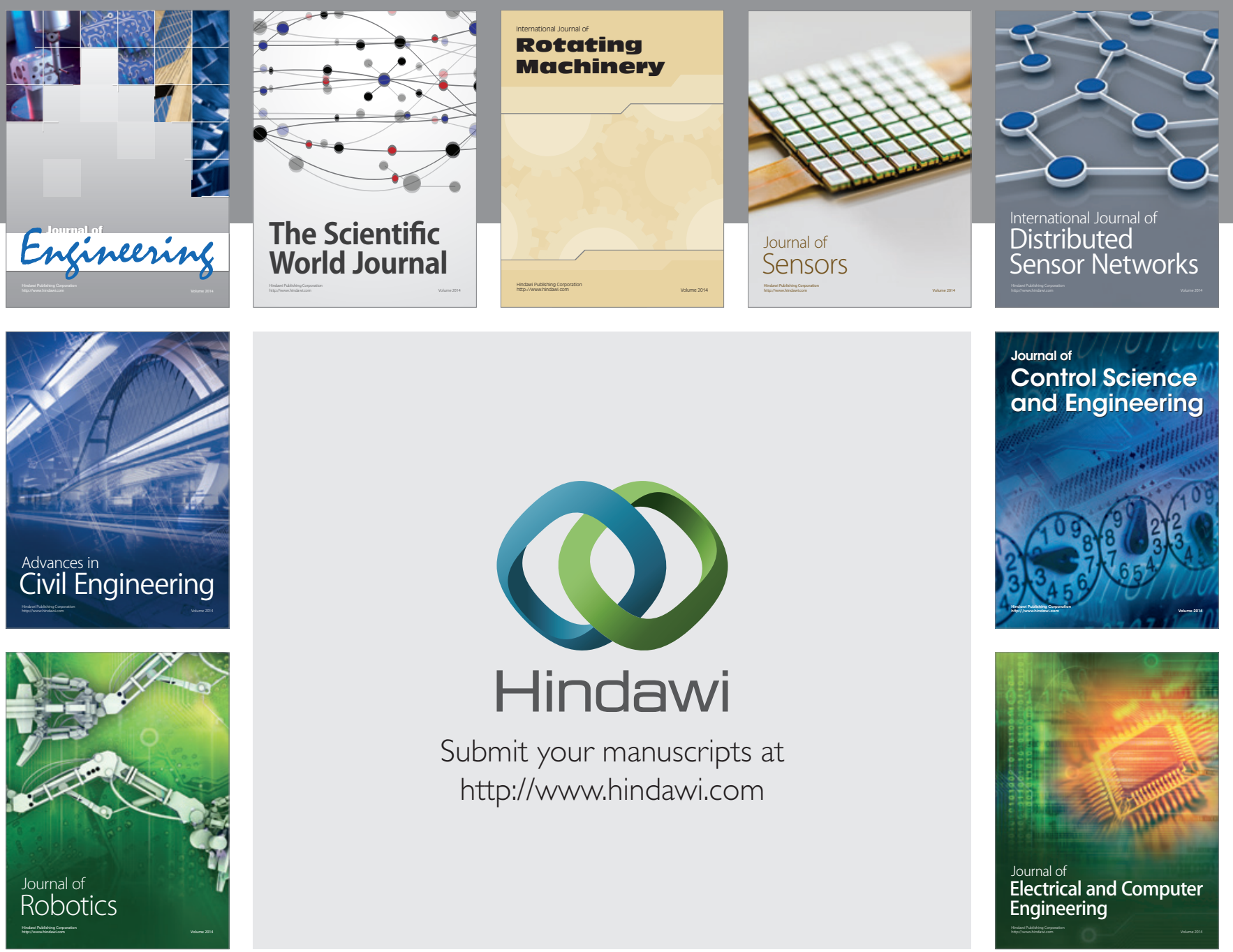

Submit your manuscripts at

http://www.hindawi.com
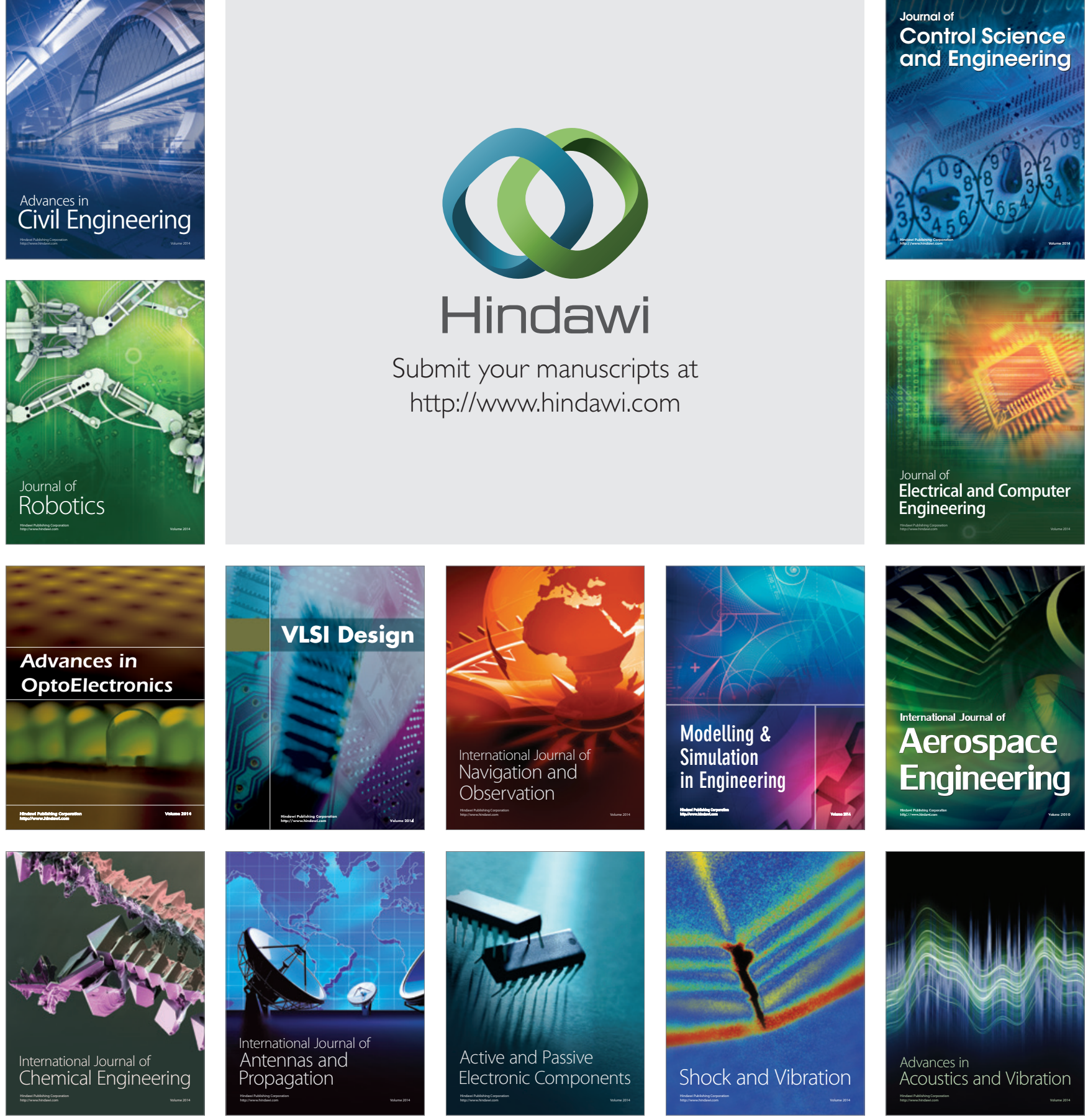